TITLE:

\title{
Three-dimensional traveling waves in a square duct
}

$\operatorname{AUTHOR}(S)$ :

Wedin, Hakan; Bottaro, Alessandro; Nagata, Masato

\section{CITATION:}

Wedin, Hakan ... [et al]. Three-dimensional traveling waves in a square duct. PHYSICAL REVIEW E 2009, 79(6): 065305.

ISSUE DATE:

2009-06

URL:

http://hdl.handle.net/2433/109851

RIGHT:

(C) 2009 The American Physical Society 
PHYSICAL REVIEW E 79, 065305(R) (2009)

\title{
Three-dimensional traveling waves in a square duct
}

\author{
Håkan Wedin and Alessandro Bottaro \\ DICAT, University of Genova, Via Montallegro 1, 16145 Genova, Italy
}

Masato Nagata

Department of Aeronautics and Astronautics, Graduate School of Engineering, Kyoto University, Kyoto 606-8501, Japan

(Received 30 January 2009; revised manuscript received 13 April 2009; published 18 June 2009)

\begin{abstract}
A nonlinear streamwise traveling-wave solution is obtained by homotopy for square duct flow. For a particular symmetry of the perturbations, this wave comes into existence at about $\operatorname{Re}_{b}=600$ (based on half-duct width and bulk speed) for a streamwise wave number $\alpha=0.85$. The resulting four-vortex mean flow resembles the transitional flow structures observed in previous simulations.
\end{abstract}

DOI: 10.1103/PhysRevE.79.065305

PACS number(s): 47.50.Gj, 47.27.De, 47.27.N-, 47.27.Cn

\section{INTRODUCTION}

The study of transition to turbulence in a square duct flow started with Nikuradse in 1926 [1]. Under turbulent conditions, a secondary mean flow was observed in the shape of an eight-vortex state with two vortices near each corner of the cross section [2-4]. Secondary flow refers here to the timeaveraged corner vortices produced by anisotropic turbulent fluctuations. To characterize the flow through a duct, one can use the bulk Reynolds number $\operatorname{Re}_{b}=\hat{U}_{b} \hat{b} / \hat{\nu}$, where $\hat{U}_{b}, \hat{b}$, and $\hat{\nu}$ are the bulk speed, the half-duct height, and the kinematic viscosity, respectively. For a square duct (i.e., aspect ratio $A=1$ ), the flow is linearly stable at all values of $\mathrm{Re}_{b}$; it becomes linearly unstable at $A \sim 3.2$ for $\operatorname{Re}_{b} \sim 23000$ [5]. For a square duct, all disturbances decay monotonically below $\mathrm{Re}_{b}^{(e)}=38$ [6]. Furthermore, numerical simulations indicate that the lower limit for transition in a square duct is between 865 and $1077[7,8]$. Fully developed turbulence is observed beyond $\mathrm{Re}_{b}=2000$.

Previous research on square duct flow has mostly focused on the mechanism generating the secondary flows under turbulent conditions. Recently, it has been shown that the flow at transitional Reynolds numbers can feature a four-vortex state; when averaging over a long-time interval, the wellknown eight-vortex state emerges out of the instantaneous four-vortex structure. In the turbulent regime, the mean flow was shown to be always in the eight-vortex state [7]. The optimal disturbance (i.e., the initial flow structure inducing the largest linear transient amplification) in a square duct is either a streamwise-independent two-vortex [6,9] or 4-vortex state [6]. These structures are very robust when used as small amplitude initial conditions for nonlinear simulations and produce flows which inevitably revert slowly to the laminar state. Transition to turbulence is easily initiated only when streamwise-dependent traveling waves (TWs) are used to initialize the simulations [6].

Canonical laminar flows such as square duct or pipe flow are linearly stable; hence, transition cannot be viewed as a step-by-step series of supercritical bifurcations from a known laminar state. The onset of chaos is believed to be related to the emergence of unstable equilibrium solutions, such as TWs or steady states in a frame moving with the wave velocity, caused by a perturbation of finite amplitude that takes the flow out of the basin of attraction of the laminar state. The TWs are disconnected from the laminar solution for linearly stable flows and their emergence provides a lower bound $\operatorname{Re}_{b}^{(\mathrm{TW})}$ in-phase space. They are believed to constitute the skeleton around which a time-dependent trajectory inphase space is organized [10]. The fact that TWs are unstable explains why a flow never settles onto such states. The very first nonlinear equilibrium solutions were discovered numerically by Nagata [11] in 1990 for plane Couette flow. Later, for plane Poiseuille flow solutions were found down to $\operatorname{Re}_{b}^{(\mathrm{TW})} \sim 1000[12,13]$. Earlier numerical and experimental studies have identified nonlinear TW solutions in pipe flow $[10,14,15]$. Later on, additional TW states were discovered for pipe flow [16,17], asymmetric solutions [18], and relative periodic orbits [19]. The review by Eckhardt et al. [20] sums up current views on the transition to turbulence in pipe flow and Kerswell [21] discussed the TW and their implications in the dynamics of the transition.

No nonlinear TW solutions have yet been reported for the square duct flow, although numerical evidence $[7,8]$ seems to indicate the existence of a four-vortex mean flow in the transitional regime.

\section{DEFINITIONS}

The isothermal incompressible flow through a square duct is considered. The dimensional Cartesian coordinates employed are $\hat{x}, \hat{y}$, and $\hat{z}$ and define the streamwise and crossstream directions, respectively. The corresponding unit vectors are $\mathbf{i}, \mathbf{j}$ and $\mathbf{k}$, with $\hat{\boldsymbol{u}}=\hat{u} \mathbf{i}+\hat{v} \mathbf{j}+\hat{w} \mathbf{k}$ as the velocity vector, $\hat{\rho}$ as the fluid density, $\hat{p}$ as the pressure, and $\hat{\nu}$ as the kinematic viscosity. The flow is forced by a constant applied pressure gradient $\overline{\hat{P}}_{x}$. The fluid is confined by four walls and the domain is $-\hat{b} \leq \hat{y}, \hat{z} \leq \hat{b}$. The duct is infinite in the $\hat{x}$ direction; hence, we impose periodicity in $\hat{x}$ and no slip at the walls, i.e., $\hat{\boldsymbol{u}}(\hat{t}, \hat{x}, \hat{y}, \hat{z})=\hat{\boldsymbol{u}}\left(\hat{t}, \hat{x}+\hat{L}_{x}, \hat{y}, \hat{z}\right)$ and $\hat{\boldsymbol{u}}(\hat{t}, \hat{x}, \hat{y}= \pm \hat{b}, \hat{z})$ $=\hat{\boldsymbol{u}}(\hat{t}, \hat{x}, \hat{y}, \hat{z}= \pm \hat{b})=\mathbf{0}$. The governing equations are nondimensionalized by using the following scales for length, time, velocity, and pressure: $\hat{b}, \hat{b} / \overline{\hat{U}}_{\max }, \overline{\hat{U}}_{\max }, \hat{\rho} \overline{\hat{U}}_{\max }^{2}$, where $\overline{\hat{U}}_{\max }$ is the maximum (centerline) laminar streamwise velocity of the flow. The mean velocity over $x$ is defined by an overbar, i.e., 


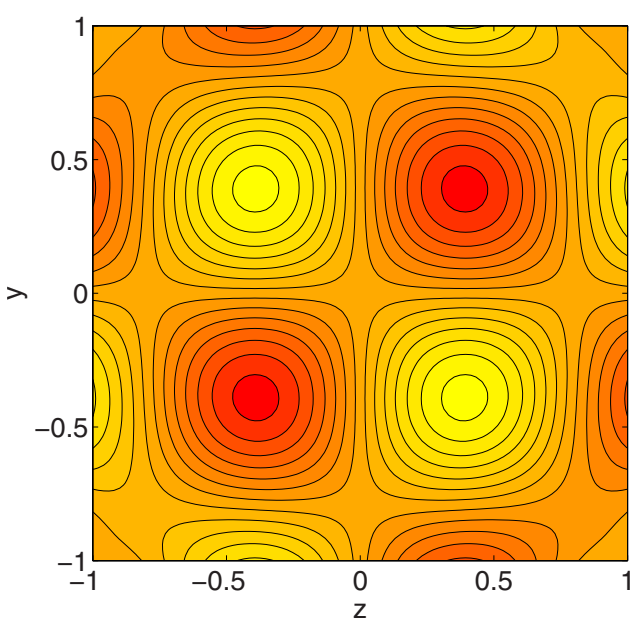

FIG. 1. (Color online) The forcing function $f(y, z)$ used for forcing the mean four-vortex flow. The color coding goes from most negative (dark) to most positive (light).

$\bar{u}=\frac{1}{L_{x}} \int_{x} u d x$ where $u$ represents the dimensionless streamwise velocity component. The bulk velocity is defined by $U_{b}$ $=\frac{1}{4} \int_{-1}^{+1} \int_{-1}^{+1} \bar{u} d y d z$. The Reynolds number Re and the bulk Reynolds number $\operatorname{Re}_{b}$ are defined as $\operatorname{Re}=\overline{\hat{U}}_{\max } \hat{b} / \hat{\nu}$ and $\operatorname{Re}_{b}$ $=\hat{U}_{b} \hat{b} / \hat{\nu}$. The latter definition is used to relate the solutions to turbulent data. The skin friction is defined as $\Lambda=8 \hat{u}_{\tau}^{2} / \hat{U}_{b}^{2}$, where $\hat{u}_{\tau}^{2}=(-2 \hat{b} / 4 \hat{\rho}) \overline{\hat{P}}_{x}$. The cross-sectional energy is defined as $E_{U}=\frac{1}{4} \int_{-1}^{+1} \int_{-1}^{+1} \frac{1}{2}\left(\bar{u}^{2}+\bar{v}^{2}+\bar{w}^{2}\right) d y d z$. We impose a perturbation $\boldsymbol{u}^{\prime}=\left(u^{\prime}, v^{\prime}, w^{\prime}\right)$ and $p^{\prime}$ on the laminar base flow $\bar{U}(y, z)$ and $\bar{P}_{x}$. The nondimensional governing equations for the perturbations are thus

$$
\left(\partial_{t}+\bar{U} \partial_{x}+\boldsymbol{u}^{\prime} \cdot \nabla-\frac{1}{\operatorname{Re}} \nabla^{2}\right) \boldsymbol{u}^{\prime}+\mathbf{i}\left(v^{\prime} \partial_{y}+w^{\prime} \partial_{z}\right) \bar{U}+\nabla p^{\prime}=\mathbf{0}
$$

and $\boldsymbol{\nabla} \cdot \boldsymbol{u}^{\prime}=0$. The disturbance is expressed as:

$$
\begin{aligned}
\boldsymbol{u}^{\prime} & =\sum_{b=-N_{x}}^{N_{x}} \widetilde{\boldsymbol{u}}^{(b)}(y, z) e^{I b \alpha(x-c t)} \\
& =\sum_{b=-N_{x}}^{N_{x}} \sum_{i=0}^{N_{y}} \sum_{j=0}^{N_{z}} \hat{\boldsymbol{u}}_{b i j} \phi_{i}(y) \phi_{j}(z) e^{I b \alpha(x-c t)},
\end{aligned}
$$

where $I=\sqrt{-1}, \alpha$ is the streamwise wave number and $c$ is the wave speed. By considering a frame of reference moving with velocity $c$, a time-independent problem with $-c \partial_{x}$ in place of $\partial_{t}$ is found in Eq. (1). The functions $\phi_{n}$ are modified Chebyshev polynomials satisfying (depending on the velocity component) either homogeneous Dirichlet boundary conditions or homogeneous Dirichlet and Neumann boundary conditions. The steady laminar flow velocity $\boldsymbol{u}=\bar{U}(y, z) \mathbf{i}$ satisfies $\nabla^{2} \bar{U}=\operatorname{Re} \bar{P}_{x}$ with no-slip conditions at the four walls and $\bar{U}(0,0)=1$ for a given $\bar{P}_{x}$.

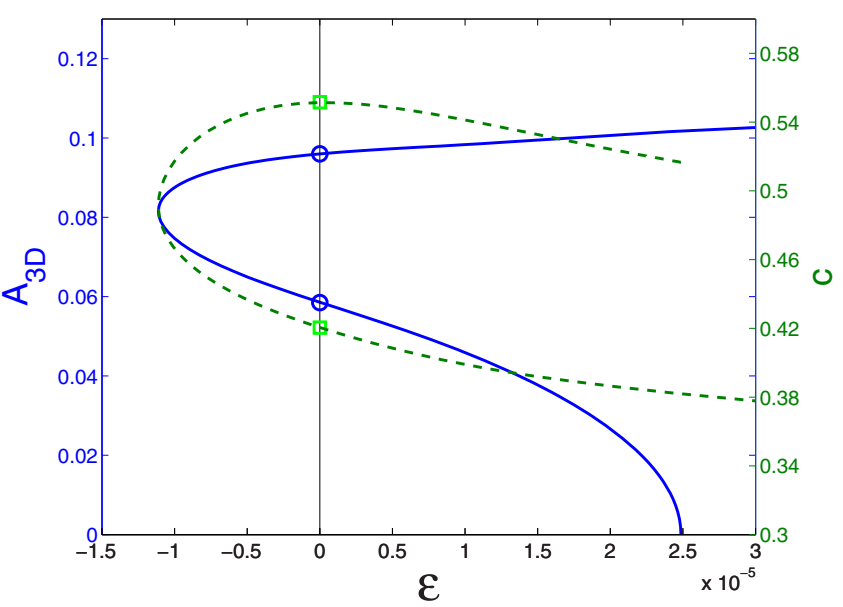

FIG. 2. (Color online) The continuation branch for $\mathrm{Re}=3000$ and $\alpha=1.5$. The curve is traced out with a resolution $\left(N_{x}, N_{y}, N_{z}\right)$ $=(4,15,15)$, which is acceptable. The amplitude of the TW is defined as $A_{3 D}=\sqrt{\sum_{j=0}^{N Z}\left|\hat{u}_{10 j}\right|^{2}+\left|\hat{v}_{10 j}\right|^{2}+\left|\hat{w}_{10 j}\right|^{2}}$, with $\hat{\boldsymbol{u}}_{10 j}$ as the solution coefficients of Eq. (2). The right vertical axis shows the phase velocity $c$ (dashed) of the wave scaled by $\overline{\hat{U}}_{\max }$.

\section{HOMOTOPY APPROACH}

Our search for nonlinear solutions is based on the study by Wedin et al. [22], which used the self-sustaining process (SSP) to identify approximate solutions to the Navier-Stokes equations. For the nonlinear study, we arrive at a system of equations for the wavy part $v^{\prime}$ and $w^{\prime}$ and the mean flow $\widetilde{u}^{(0)}(y, z)$ (the streaks) and $\widetilde{\psi}^{(0)}(y, z)$ (the stream function). The superscript of the mean flow refers to the zeroth streamwise mode [cf. Eq. (2)]. There are various symmetries in this system and we have imposed both $\boldsymbol{S}$ and $\boldsymbol{Z}$ below,

$$
\begin{gathered}
S:(x, y, z) \rightarrow(x+\pi / \alpha,-y, z), \quad(u, v, w, p) \rightarrow(u,-v, w, p), \\
Z:(x, y, z) \rightarrow(x, y,-z), \quad(u, v, w, p) \rightarrow(u, v,-w, p) .
\end{gathered}
$$

To find nonlinear solutions of the Navier-Stokes equations, the SSP is converted into a continuation procedure by involving a forcing function $f(y, z)$ with an amplitude $\epsilon$ $[13-15,23,24]$. Figure 1 shows the $f(y, z)$ that forces the nonlinear system. We begin the search for nonlinear solutions by establishing a bifurcation point by forcing streaks $\widetilde{u}^{(0)}(y, z)$ and the stream function $\widetilde{\psi}^{(0)}(y, z)$ using $f(y, z)$, without the wavy part, i.e.,

$$
\begin{gathered}
\epsilon f(y, z)=\frac{1}{\operatorname{Re}} \nabla^{4} \widetilde{\psi}^{(0)}+\mathcal{N}\left(\widetilde{\psi}^{(0)}\right), \\
\left(\widetilde{\psi}_{z}^{(0)} \partial_{y}-\widetilde{\psi}_{y}^{(0)} \partial_{z}-\frac{1}{\operatorname{Re}} \nabla^{2}\right) \widetilde{u}^{(0)}=\left(-\widetilde{\psi}_{z}^{(0)} \partial_{y}+\widetilde{\psi}_{y}^{(0)} \partial_{z}\right) \bar{U},
\end{gathered}
$$

with $\mathcal{N}$ as the nonlinear terms. A linear stability analysis of the basic flow of the forced system, i.e., $\left[\bar{U}+\widetilde{u}^{(0)}, \widetilde{\psi}^{(0)}\right]$, is then performed. Typically, a neutrally stable mode is found near that which was identified in [22] from where we can start searching for nonlinear solutions. The initial conditions in parameter space are the same as in Wedin et al. [22] 
TABLE I. Properties of the TW solution at the turning point for two truncations. The streamwise velocity is defined as $u^{\prime}$ and the roll structure is defined as $\boldsymbol{u}_{\perp}^{\prime}=v^{\prime} \boldsymbol{j}+w^{\prime} \boldsymbol{k}$; DOF means degrees of freedom.

\begin{tabular}{cccccccc}
\hline \hline$\alpha$ & $\min \left[\mathrm{Re}_{b}\right]$ & $\Lambda$ & $c$ & $\left\|\overline{\boldsymbol{u}^{\prime}}{ }_{\perp}\right\|$ & $\left\|\overline{u^{\prime}}\right\|$ & Truncation & DOF \\
\hline 0.85 & 598.2 & 0.074 & 0.475 & 0.021 & 0.570 & $(7,20,20)$ & 13231 \\
0.85 & 596.1 & 0.074 & 0.477 & 0.021 & 0.569 & $(4,15,15)$ & 4609 \\
\hline \hline
\end{tabular}

( $\operatorname{Re}=3000)$, with either $\alpha=1.5$ or $\alpha=0.282$ and $\operatorname{Re}=5000$ with $\alpha=3.415$, with the aim to reduce $\epsilon$ to zero while maintaining a nonlinear state. The initial conditions at $\alpha=0.282$ and $\alpha=3.415$ did not cross $\epsilon=0$ in the course of the iterations, which explains why the direct numerical simulations in [22] relaminarized. Therefore, focus is put on the solution at $\alpha=1.5$. This initial condition leads to the continuation branch in Fig. 2. The two solutions at $\epsilon=0$ are used as starting points for mapping out the solutions in parameter space.

\section{SEARCH FOR NONLINEAR SOLUTIONS}

The point in $\operatorname{Re}_{b}(\alpha)$ where the TW emerges is located by a search over $\alpha$. The absolute minimum $\left[\operatorname{Re}_{b}\right]=598.2$ occurs
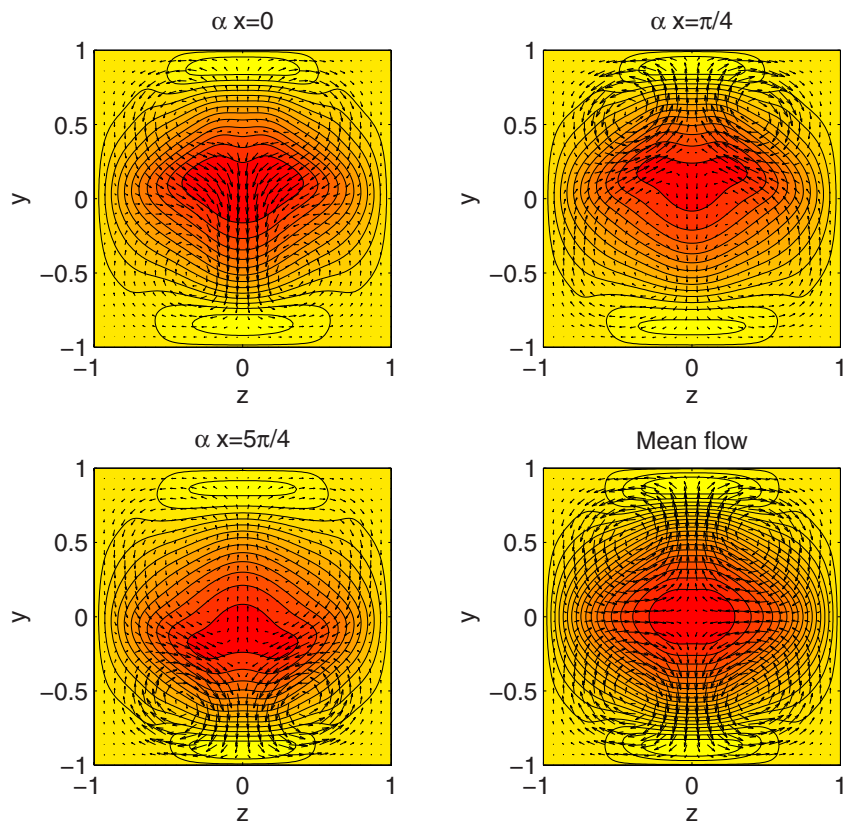

FIG. 3. (Color online) The disturbance velocity field at the turning point (see Table I) at three stations in $x$ and the mean flow. The contour levels represent the streamwise velocity $u^{\prime}$ and range between the minimum and the maximum of $u^{\prime}$ of the chosen stations in $x$ or -0.586 to 0.126 in steps of 0.05 . An online video provides a clear visualization of the TW [25]. The bottom right figure shows the mean flow in the shape of a four-vortex structure, similar to that observed in transitional duct flow [7]. The contour levels represent the mean streamwise velocity $\overline{u^{\prime}}$ and range between the minimum and the maximum of $\overline{u^{\prime}},-0.570$ to 0.099 in steps of 0.032 . Compare with Figs. 3(a) and 3(b) of Uhlmann et al. [7]. The color coding goes from most negative (dark) to most positive (light). The arrows represent the cross-stream velocity components. for $\alpha=0.85$. Table I sums up the properties of the solution found.

The structure of the nonlinear solution is shown in Fig. 3. In the streamwise direction, the rolls (represented by arrows) move up and down in the $y$ direction with a pronounced swirl next to $y=+1$ or $y=-1$ depending on the streamwise position $x$. The streamwise high-speed streaks (light yellow contour levels online) move about little with $x$, as observed also in pipe flow [14], whereas the low-speed streaks (light red online) move rapidly in the center.

This streamwise behavior is similar to that of the socalled N1-solution discovered by Pringle et al. [17]. Averaging over $x$ results in the four-vortex state shown in Fig. 3 with one vortex in each quadrant, similar to the mean state observed in direct numerical simulations (DNS) of transitional square duct flow [7]. It is clear that the solution rotated by $90^{\circ}$ also exists. DNS display solutions which alternate in time, first with the vortex upwash near the vertical walls and

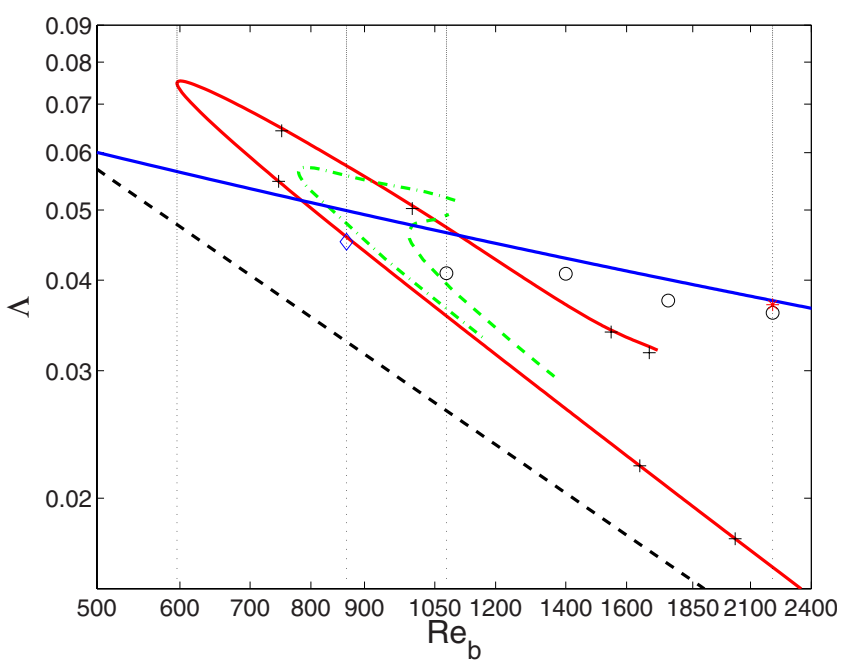

FIG. 4. (Color online) The skin friction $\Lambda$ (red solid curve) as a function of $\operatorname{Re}_{b}$ for $\alpha=0.85$ and resolution $(4,15,15)$. The plus (+) represent results with resolution $(6,20,20)$, showing good agreement with the coarser grid simulations. The dashed black line represents the laminar flow and the solid blue line turbulent experimental data [26] approximated by $\Lambda^{-0.5}=2 \log _{10}\left[2.25 \operatorname{Re}_{b} \Lambda^{0.5}\right]$. The two dotted vertical lines at $\operatorname{Re}_{b}=865$ and 1077 correspond to the transitional limit according to Biau and Bottaro [8] (blue diamond) and Uhlmann et al. [7] (black empty circle; see also the three additional points shown with empty circles of the same authors for larger $\operatorname{Re}_{b}$ ). The red asterisk (*) at $\mathrm{Re}_{b}=2205$ is the result from Gavrilakis [2]. To see the effect of periodicity, pieces of the curves for $\alpha=1.5$ and 2.0 have been added (in green, respectively, with dash-dotted and dashed lines). 
then near the horizontal walls. The skin friction $\Lambda$ of this TW is shown in Fig. 4 together with the values of the laminar flow, experimental data on turbulence [26], and results from simulations $[2,7,8]$. We observe a fairly good match with the simulations $[7,8]$ and, in particular, the portion of the lower branch computed for $\alpha=0.85$ almost overlaps onto the value of Biau and Bottaro [8], whereas the lower branch for $\alpha=2$ approaches the transitional limit identified by Uhlmann et al. [7]. The difference between the transitional limits found by these two groups must be ascribed to the different choice of computational domain length. We have also compared the value of the streamwise averaged energy of the simulation of the transition process by Biau et al. [6] with the value found here. At $\operatorname{Re}_{b}=1042$ in the turbulent regime, the average value of $E_{U}$ was found to be 0.0594 in [6], whereas here $E_{U}$ varies between 0.0613 (for $\alpha=2$ ) and 0.0779 (for $\alpha=0.85$ ). The agreement is acceptable and supports the conjecture that the flow at the lower limit of transition spends most of its time in the vicinity of the four-vortex state identified here. Comparing with experimental data [26] and DNS [2] at high $\operatorname{Re}_{b}$, the solution identified here displays a lower skin friction on both branches for $\mathrm{Re}_{b} \gtrsim 1100$, indicating that other nonlinear TW or periodic orbit solutions are likely to play a part of the dynamics of the turbulence as $\mathrm{Re}_{b}$ increases.

\section{CONCLUSION}

A nonlinear TW solution has been discovered in a square duct down to a bulk Reynolds number of $\operatorname{Re}_{b}^{(\mathrm{TW})}=598.2$ at a streamwise wave number of $\alpha=0.85$. At transitional $\mathrm{Re}_{b}$, the value of the skin friction on the lower branch lies in the neighborhood of values observed in direct numerical simulations $[7,8]$. There must exist heteroclinic connections with other solutions; but based on the shape of the secondary flow, the values of skin friction, and the streamwise averaged mean energy, we conjecture that during transition the flow spends most of the time in the neighborhood of the state found here. Our solution is similar to a corresponding TW state identified in the circular pipe [17] highlighting the similarity between these two flow cases. Other solutions with different symmetries are currently being investigated to provide a complete picture of available states.

\section{ACKNOWLEDGMENTS}

The financial support of a EU Marie-Curie Intra-European Action (Grant No. PIEF-GA-2008-220201) and of the Italian Ministry of University and Research (Grant No. PRIN $2007-$ 4JXHH2-002) is gratefully acknowledged. H.W. wishes also to acknowledge the support from the Blanceflor Boncompagni-Ludovisi Foundation.
[1] J. Nikuradse, Ph.D. thesis, VDI-Forschungsheft 281, Berlin, 1926.

[2] S. Gavrilakis, J. Fluid Mech. 244, 101 (1992).

[3] F. Gessner, J. Fluid Mech. 58, 1 (1973).

[4] A. Huser and S. Biringen, J. Fluid Mech. 257, 65 (1993).

[5] T. Tatsumi and T. Yoshimura, J. Fluid Mech. 212, 437 (1990).

[6] D. Biau, H. Soueid, and A. Bottaro, J. Fluid Mech. 596, 133 (2008).

[7] M. Uhlmann, A. Pinelli, G. Kawahara, and A. Sekimoto, J. Fluid Mech. 588, 153 (2007).

[8] D. Biau and A. Bottaro, Philos. Trans. R. Soc. London, Ser. A 367, 529 (2009).

[9] B. Galletti and A. Bottaro, J. Fluid Mech. 512, 85 (2004).

[10] B. Hof, C. van Doorne, J. Westerweel, F. Nieuwstadt, H. Faisst, B. Eckhardt, H. Wedin, R. Kerswell, and F. Waleffe, Science 305, 1594 (2004).

[11] M. Nagata, J. Fluid Mech. 217, 519 (1990).

[12] U. Ehrenstein and W. Koch, J. Fluid Mech. 228, 111 (1991).

[13] F. Waleffe, Phys. Fluids 15, 1517 (2003).

[14] H. Wedin and R. Kerswell, J. Fluid Mech. 508, 333 (2004).
[15] H. Faisst and B. Eckhardt, Phys. Rev. Lett. 91, 224502 (2003).

[16] Y. Duguet, A. Willis, and R. Kerswell, J. Fluid Mech. 613, 255 (2008).

[17] C. Pringle, Y. Duguet, and R. Kerswell, Philos. Trans. R. Soc. London, Ser. A 367, 457 (2009).

[18] C. C. T. Pringle and R. R. Kerswell, Phys. Rev. Lett. 99, 074502 (2007).

[19] Y. Duguet, C. Pringle, and R. Kerswell, Phys. Fluids 20, 114102 (2008).

[20] B. H. B. Eckhardt, T. M. Schneider, and J. Westerweel, Annu. Rev. Fluid Mech. 39, 447 (2007).

[21] R. Kerswell, Nonlinearity 18, R17 (2005).

[22] H. Wedin, D. Biau, A. Bottaro, and M. Nagata, Phys. Fluids 20, 094105 (2008).

[23] F. Waleffe, Phys. Fluids 9, 883 (1997).

[24] F. Waleffe, Phys. Rev. Lett. 81, 4140 (1998).

[25] See EPAPS Document No. E-PLEEE8-79-R16906 for a visualization of the traveling wave. For more information on EPAPS, see http://www.aip.org/pubservs/epaps.html.

[26] O. Jones, ASME Trans. J. Fluids Eng. 98, 173 (1976). 